\title{
ALGUNAS CONSIDERACIONES ACERCA DE LA FAMILIA IRIDACEAE EN EL VALLE DE MEXICO'
}

\author{
Graciela Calderon de Rzedowski \\ Instituto de Ecología \\ Centro Regional del Bajio \\ Apartado postal 386 \\ 61600 Pátzcuaro, Mich. México
}

\begin{abstract}
RESUMEN
Como resultado de la revisión realizada para el volumen III de la "Flora Fanerogámica del Valle de México", se reconocen para esta región los siguientes taxa de la familia Iridaceae: Nemastylis tenuis (Herb.) Wats., Orthrosanthus chimboracensis var. exsertus Foster, Sisyrinchium angustissimum (Rob. \& Greenm.) Greenm. \& Thomps., S. arizonicum Rothr., S. cernuum (Bicknell) Kearney, S. convolutum Nocca, S. conzattii Calderón \& Rzedowski, S. quadrangulatum Klatt, S. scabrum Schl. \& Cham., S. schaffineri Wats., S. tenuifolium H. \& B. ex Willd. S. tolucense Peyr., Tigridia alpestris Molseed ssp. alpestris, T. martinezii Calderón, T. multiflora (Baker) Ravenna, T. pavonia (L.f.) DC., T. vanhouttei ssp. roldanii Molseed, T. vanhouttei Roezl ssp. vanhouttei, T. violacea Schiede ex Schl. y Tritonia crocosmifflora Nichols. De este conjunto: Sisyrinchium conzattii y Tigridia martinezii se descubrieron como especies nuevas para la ciencia; S. arizonicum, S. cernuum, Tigridia multiflora, T. violacea y Tritonia crocosmiiflora se citan por primera vez para la flora de la región; $S$. tolucense es el nombre que se aplica para plantas conocidas con anterioridad, pero que se habian identificado por lo general de otra manera; Nemastylis caerulescens Greenm. y Rigidella flammea Lindl., mencionadas por otros autores, se excluyen por no haber pruebas seguras de su existencia en el área de estudio.
\end{abstract}

\section{ABSTRACT}

In the treatment of the family Iridaceae for the "Flora Fanerogámica del Valle de México" the following taxa area recognized: Nemastylis tenuis (Herb.) Wats., Orthrosanthus chimboracensis var. exsertus Foster, Sisyrinchium angustissimum (Rob. \& Greenm.) Greenm. \& Thomps., S. arizonicum Rothr., S. cernuum (Bicknell) Kearney, S. convolutum Nocca, S. conzattii Calderón \& Rzedowski, S. quadrangulatum Klatt, S. scabrum Schl. \& Cham., S. schaffneri Wats., S. tenuifolium H. \& B. ex Willd., S. tolucense Peyr., Tigridia alpestris Molseed ssp. alpestris, T. martinezii Calderón, T. multiflora (Baker) Ravenna, T. pavonia (L.f.) DC., T. vanhouttei ssp. roldanii Molseed, T. vanhouttei Roezl ssp. vanhouttei, $T$. violacea Schiede ex Schl. y Tritonia crocosmiiflora Nichols. Two taxa: Sisyrinchium conzattii and Tigridia martinezii were discovered as new species; S. arizonicum, S. cernuum, Tigridia multiflora, T. violacea and Tritonia crocosmiiflora are cited for the first time as members of the local flora; $S$. tolucense is applied to plants previously known from the area, but otherwise identified; Nemastylis caerulescens and Rigidella flammea are excluded, because no definite proofs were found of their existence in the area.

\section{INTRODUCCION}

Aún antes de comenzar con el estudio de la familia Iridaceae para el volumen III de la

\footnotetext{
- Trabajo realizado con apoyo del Centro de Investigación y Desarrollo del Estado de Michoacán y del Consejo Nacional de Ciencia y Tecnología.
} 
"Flora Fanerogámica del Valle de México", se podía prever que iban a presentarse serias dificultades dada la complejidad de este grupo, en especial en lo que se refiere a los géneros Sisyrinchium y Tigridia. Se conjeturaban asimismo cambios y adiciones que hacer con respecto a listados y planteamientos precedentes.

A lo anterior se agregó el problema de que los ejemplares de Iridáceas de México, pertenecientes al Herbario de la Escuela Nacional de Ciencias Biológicas, del Instituto Politécnico Nacional, se habian prestado (en 1974) y aunque desde febrero de 1981 se ha estado solicitando su devolución, no ha habido manera de que este material sea reintegrado. En tal situación ha sido necesaria una especial dedicación en los últimos 3 años para la localización y colecta intensiva de Iridáceas del Valle de México, lo cual tuvo la ventaja de poder hacer anotaciones apropiadas en el campo y sobre material fresco, que muchas veces son importantes para la mejor interpretación de los miembros de esta familia.

Por otro lado, se contó con los préstamos de ejemplares de herbarios nacionales y extranjeros cuyas siglas se mencionan a continuación: A, CAS, CHAPA, DS, ENEPI, G, GH, MEXU, MO, MSC, NY, US y WISC, que, junto con los reunidos para ENCB, dieron la oportunidad de revisar más de 2000 especímenes secos, lo que ha sido de una enorme utilidad.

\section{ANTECEDENTES}

Aunque en diferentes épocas varios autores han registrado la existencia de miembros de esta familia en la región estudiada, el único intento de integración son las contribuciones de Eizi Matuda, quien publicó en 1962 "Las Iridáceas del Valle de México y sus alrededores" y en 1964 , en términos semejantes, "Las Iridáceas del Estado de México". Sin embargo, a poco más de 20 años de distancia, han aparecido varios trabajos importantes, sobre todo la monografía de Tigridia de Molseed (1970), lo que unido a nuevas e intensas exploraciones y colectas en el área, ha determinado la necesidad de introducir algunas modificaciones a las inferencias de Matuda.

\section{RESULTADOS}

A continuación se discuten, por orden alfabético de géneros y especies, las conclusiones alcanzadas en esta revisión, haciendo referencia a lo ya mencionado para el Valle en publicaciones previas.

-Nemastylis caerulescens Greenm. ( $N$. tenuis var. caerulescens (Greenm.) Foster).Bajo este nombre Matuda (1962, pág. 161 y fig. 3; 1964, pág. 8 y fig. 3) y Sánchez (1969, pág. 111), han considerado una planta del género Tigridia, probablemente T. tepoztlana Ravenna, especie al parecer endémica de las cercanías de Cuernavaca, Morelos. Según Molseed (op.cit., pág. 62), la colección tipo de esta última (Pringle 8436) fue equivocadamente distribuida como N. caerulescens.

- Nemastylis tenuis (Herb.) Wats.- Especie ampliamente distribuida en la parte baja del Valle de México, sobre todo en pastizales. Foster (1945, pág. 40), Matuda (1962, pág. 161; 1964, pág. 6) y Sánchez (op. cit., pag. 111), citan también de la región a $N$. tenuis var. nana (Wats.) Foster, diferenciándola por el tamaño reducido y flores de color azul pálido; sin 
embargo, en vista de existir en nuestra área poblaciones con características intermedias entre esta variedad y la típica, se ha preferido considerarla sólo como un extremo en la variación. Mención aparte merecen especímenes de talla baja y flores blancas colectados entre Pachuca y Real del Monte. En la descripción original de $N$. nana Wats., se dice que el color de la flor es "blanco-verdoso", aunque Foster (loc. cit.) achaca tal condición a efectos del secado. Estos ejemplares de la Sierra de Pachuca se incluyen también dentro de $N$. tenuis, ya que en los demás caracteres no difieren mayormente de tal especie.

-Orthrosanthus chimboracensis var. exsertus Foster ( $O$. monadelphus ssp. exsertus (Foster) Ravenna).- Escasa en nuestra zona de estudio, es una herbácea robusta de visiosas flores azules a blancas. Ravenna (1965, pág. 317) considera las plantas norteamericanas de este conjunto como 0 . monadelphus, pero es preferible el criterio más inclusivo de Steyermark (1948, pág. 17), quien reconoce una sola especie distribuida desde Perú hasta México.

-Rigidella flammea Lindl.- Hemsley (1879-1888, pág. 327) refiere esta especie de "...Valley of Mexico (Bourgeau, 740)", sin embargo ya Reiche (1926, pág. 263) comenta que "... $R$. flammea está citada del Valle Central, pero probablemente por equivocación". Se trata de una planta muy vistosa, difícil de pasar inadvertida, que debe excluirse de la flora del Valle de México, ya que, como lo establece Cruden (1971, pág. 224), su área de distribución se encuentra restringida a la zona de Mil Cumbres, en el noreste del estado de Michoacán.

SISYRINCHUIM constituye un género americano de regiones templadas y tropicales para el que se calculan entre 30 y 125 especies, habiendo autores que francamente admiten un número "incierto". Esto último sobre todo, da idea de la dificultad del grupo y de la necesidad de un estudio crítico actualizado. La presente revisión permitió reconocer 10 especies para el Valle de México, haciendo la aclaración de que en muchos casos queda la duda en la interpretación de los límites reales de una especie dada y en otros casos en lo que se refiere a la aplicación correcta de los nombres.

-S. angustissimum (Rob. \& Greenm.) Greenm. \& Thomps.- Planta perenne, más bien alta (hasta de $1 \mathrm{~m}$ ) y delicada, con el tallo flexuoso, ramificado; de distribución restringida a las delegaciones de Contreras y Tlalpan. No siempre es de fácil separación de individuos altos y esbeltos de la muy variable $S$. tenuifolium.

-S. arizonicum Rothr.- Se aplica de manera algo tentativa este nombre a plantas de porte robusto y flores relativamente grandes ( 3.5 a $4 \mathrm{~cm}$ de diámetro), que aparentemente no habían sido registradas para la zona de estudio con anterioridad. Se le conoce de los municipios y delegaciones de Huehuetoca, Coyotepec, Naucalpan, Huixquilucan, Tlalpan, Tláhuac, Ixtapaluca y Tlalmanalco, de altitudes entre 2400 y $3400 \mathrm{~m}$; escasa, aunque localmente abundante en matorrales, pastizales o bosques de pino o encino, a menudo sobre peñas o en lugares de pendiente pronunciada. Pertenece a un complejo de especies no bien comprendido todavía, en el cual se encuentran poblaciones de porte semejante que habitan en diferentes regiones de la República, así como en Arizona y Nuevo México. Algunas se cultivan localmente por la hermosura de sus flores.

-S. cernuum (Bicknell) Kearney.- Corresponden aquí plantas poco conspicuas, razón por la que posiblemente habían pasado desapercibidas; así, en el Valie de México suelen no ser mayores de $10 \mathrm{~cm}$ de alto, tienen flores pequeñas (hasta de $5 \mathrm{~mm}$ de diámetro) y frutos subglobosos, colgantes sobre finos pedicelos; son escasas, aunque a veces localmente 
abundantes en manchones. Se ha colectado en los últimos años en los municipios y delegaciones de Real del Monte, Epazoyucan, Tepotzotlán, Coyotepec, Cuajimalpa y Villa A. Obregón, en altitudes entre 2750 y 3100 m, en sitios cenagosos o rocosos, en encinares o en bosquete perturbado de Juniperus con magueyes sembrados. Especie registrada del sureste de Arizona al centro de México, suele estar mejor desarrollada y representada en algunas regiones fuera de nuestra área de estudio. Pertenece al grupo que Bicknell (1900) consideró como el género Hydastylus; el mencionado autor describió en su trabajo varias especies, algunas de las cuales no parecen diferir mayormente de $S$. cernuum.

-S. convolutum Nocca.- Este nombre se ha venido usando más por tradición que por discernimiento y aquí se le acepta, por lo menos tentativamente, para un conjunto de plantas de hojas más bien anchas, dobladas sobre sí mismas, la base algo ampulosa, raíces no tuberosas y flores relativamente grandes (2 a $4 \mathrm{~cm}$ de diámetro); se ha colectado en los municipios y delegaciones de El Chico y Epazoyucan, Villa Nicolás Romero, Iturbide, Cuajimalpa, Contreras, Tlalpan y Tlalnepantla, a altitudes de 2300 , o más frecuentemente entre 2750 y $3100 \mathrm{~m}$, en lugares francamente cenagosos en medio de pastizales o bosques; a veces favorecida su presencia por el disturbio, se encuentran poblaciones cerca o entre los cultivos. Algunos ejemplares, procedentes de Lanzarote, municipio de Villa Nicolás Romero, son más robustos y algo diferentes de los demás, pero a la vez muy semejantes a unos colectados en Tlalpan y Tlalnepantla a principios de siglo e identificados bajo este nombre. Es necesario, sin embargo, un estudio crítico para delimitar si se trata de 1 o de 2 especies y el o los nombres correctos que les corresponderían.

-S. conzattii Calderón \& Rzedowski.- Se caracteriza por su aspecto esbelto y flores amarillas con el centro obscuro. Conocida en el Valle de México de los municipios de Jiquipilco, Ixtapaluca y Tlalmanalco, así como de la delegación de Cuajimalpa, en altitudes entre 3300 y $3600 \mathrm{~m}$, en bosque de Pinus hartwegii y claros adyacentes o en zacatonales. También colectada por encima de la vegetación arbórea en el Nevado de Toluca, Estado de México.

-S. quadrangulatum Klatt.- Plantas de apariencia delicada, tallo cuadrangular y fruto obpiriforme, habitantes de las altas montañas a altitudes entre 3400 y $4000 \mathrm{~m}$, en bosques de Pinus hartwegiiy en zacatonal alpino, tanto del sur del Valle de México como de Jalisco, Estado de México y Veracruz. Esta especie había sido citada de la Sierra Nevada por Beaman (1965,pág. 71).

-S. scabrum Schl. \& Cham. (S. affine Mart. \& Gal.).- Se aplica este nombre a la única especie local con flor que varía del azul al morado (en las demás la flor es amarilla), perenne, pero variable en hábito y en las dimensiones de sus diferentes partes, común y de amplia distribución geográfica y ecológica en el Valle de México. Aún cuando Johnston (1938, pág. 390) ubicó a S. scabrum como sinónimo de $S$. micranthum Cav., especie anual de lugares más calurosos, recientemente McVaugh (com. pers.) pudo comprobar que el material original de $S$. scabrumcorresponde a la especie representada en el Valle de México. Pertenece a un complejo particularmente diversificado en los Estados Unidos de América, cuyos miembros son de difícil delimitación. Con frecuencia se le ha llamado $S$. angustifolium Mill., pero esta última especie se encuentra restringida a regiones más septentrionales. Algunos ejemplares han sido identificados como $S$. tenuifolium, tal vez por error al confundir los epítetos tenuifolium y angustifolium. No queda aún clara la demarcación de $S$. scabrum de otros miembros del complejo "angustifolium". Se han colectado plantas de aspecto similar de Chihuahua a Nuevo 
León, Veracruz, Jalisco y Chiapas, pero quizás no todas pertenecen a S. scabrumy se requiere de un análisis más detallado de este grupo.

-S. schaffneri Wats.- Vegetales de hábito fino, con hojas basales solamente, tallo sin ramificar y con una sola espata terminal, fruto elipsoide, colgante sobre pedicelo filiforme; existe en los municipios y delegaciones de El Chico, Real del Monte, Epazoyucan, Iturbide, Jilotzingo, Naucalpan, Huixquilucan, Cuajimalpa, Villa A. Obregón, Contreras, Texcoco, Ixtapaluca, Tlalmanalco y Amecameca, en altitudes de 2500 y más frecuentemente entre 2800 y $3350 \mathrm{~m}$, en suelos encharcados en medio de pastizales y bosques de varios tipos, a veces localmente abundante. Reconocida como miembro de la flora del Valle desde principios de este siglo, ha sido aceptada aquí tentativamente, ya que el tipo, procedente de San Luis Potosí, representa una planta un poco diferente. Además algunos ejemplares colectados en los municipios de Epazoyucan, El Chico y Tlalmanalco, poseen características que los asemejan a S. tinctorium H.B.K., registrada desde el sur de México hasta el norte de Sudamérica. Sin embargo, existen plantas con rasgos intermedios, por lo cual es difícil decidir si se trata de 1 ó 2 entidades y cual sería el nombre correcto correspondiente.

-S. tenuifolium H. \& B. ex Willd.- Constituye la especie de Sisyrinchium mejor representada y de distribución geográfica y ecológica más amplia (alt. 2250-3800 m) en el Valle de México. Altamente variable en aspecto y características, se mantiene constante en la presencia de la pubescencia en el ovario y el fruto, aunque en ocasiones en este último se ve substituida por verrugas o púas cortas. A esta especie se refieren Reiche (1914, pág. 125), Beaman (op. cit. pág. 71), Matuda (1962, pág. 174; 1964, pág. 19) y Sánchez (op. cit. pág. 112), también bajo el nombre de S. bracteatum Greenm., con base en individuos de tallos flexuosos. De igual manera, pertenecen aquí los ejemplares de talla baja denominados a veces como $S$. tenuifolium var. seatonii Greenm. En ambos casos existen muchas poblaciones con características intermedias y mientras no haya más elementos para separarlos, se considera conveniente tratarlos como una sola entidad variable. Las flores con el centro morado obscuro prevalecen en poblaciones que habitan a mayores altitudes, pero pueden presentarse también con frecuencia en individuos de otras partes. Veáse además el comentario formulado bajo $S$. angustissimum.

-S. tolucense Peyr.- A veces confundida con la anterior por su aspecto semejante a primera vista, pero de ovario glabro como principal carácter distintivo. A menudo se le encuentra identificada como "S. tenuifolium" o "S. bracteatum", por lo que aparentemente es esta la primera vez que se menciona del Valle de México con el nombre que le corresponde. De características variables y de amplia distribución en la zona montañosa húmeda del Valle, se localiza en altitudes entre 2500 y 3550 m, en diversos habitats. S. palmeriGreenm., descrita de las cercanías de Guadalajara, al parecer no es sino una variante más robusta de la misma especie.

TIGRIDIA es un género que comprende entre 25 y 30 especies en su mayoría mexicanas, algunas se extienden a Centroamérica y una se registra de Sudamérica. Para el Valle de México se reconocen 7 taxa. Es sin duda un grupo que ofrece muchas complicaciones, pero por fortuna se cuenta con la magnífica revisión efectuada por Molseed (op. cit.). Este autor agrega la mención de $T$. alpestris Molseed ssp. alpestris y de $T$. vanhouttei ssp. roldanii Molseed, a la corta lista previa presentada por Hemsley (op. cit. págs. 326, 327), Reiche (1914, pág. 125; 1926, pág. 263), Matuda (1962, págs. 163, 164; 1964 págs. 8, 10) y Sánchez (op. cit. 
pags. 111,112 ), que se limitaban a citar a $T$. pavonia (L.f.) DC. y $T$. vanhouttei Roezl (=T. vanhouttei ssp. vanhouttel). Resta referirse a las siguientes, que habian pasado desapercibidas, probablemente debido a su escasa representación en la región.

-T. martinezii Calderón.- Esta especie, recientemente descrita, es planta rara, sólo conocida hasta la fecha de lugares rocosos poco accesibles del Cerro de las Ventanas y del Cerro Zumate, en la Sierra de Pachuca. Presenta bonitas flores de color crema manchadas de morado, de unos $2 \mathrm{~cm}$ de diámetro.

-T. multiflora (Baker) Ravenna.- En 1938 fue colectada por Lyonnet cerca de Mixcoac. En los últimos años ha sido localizada en los siguientes municipios y delegaciones: Coyotepec, Tepotzotlán, Cuajimalpa, Tlalpan, Texcoco, Temamatla y Tenango del Aire, en altitudes entre 2300 y $3000 \mathrm{~m}$, en pedregal con matorral xerófilo y en bosques de encino o de pino. Escasa, pero de amplia distribución en la República Mexicana, siguiendo ambas Sierras Madres y la Cadena Volcánica Transversal. Son plantas de alrededor de medio metro de alto con flores moradas o morado-cafés.

-T. violacea Schiede.- De unos $25 \mathrm{~cm}$ de alto, con flores de color violáceo con el centro blanquecino o amarillento. Conocida del Valle de México de la Sierra de Alcaparrosa, municipio de Tepotzotlán; vive en altitudes entre 2300 y $2500 \mathrm{~m}$, en pastizal y en claros adyacentes al bosque de encino. Se distribuye a través de la Cadena Volcánica Transversal.

- Tritonia crocosmiiflora Nichols.- Es una hierba robusta con flores grandes y vistosas de color rojo-anaranjado. Se le considera como un híbrido logrado en Francia el siglo pasado de las especies africanas Crocosmia aurea Planch. y Tritonia pottsiiBenth. Se le ha encontrado creciendo en forma espontánea en los municipios de Iturbide y Texcoco, Estado de México, en altitudes entre 2300 y $2500 \mathrm{~m}$, a orilla de arroyos en bosque de encino o como ruderal; escasa, a veces localmente abundante. En algunos países se cultiva en los jardines y en ocasiones se ha ido naturalizando. En ciertas regiones de clima cálido de México se encuentra en abundancia en el medio arvense. Se cita por primera vez como miembro de la flora del Valle de México.

\section{AGRADECIMIENTOS}

Se agradece a los encargados de los herbarios mencionados en la parte introductoria que proporcionaron préstamos de ejemplares. Al Dr. Peter H. Raven, director del Jardín Botánico de Missouri, por la ayuda en la obtención de algunos materiales bibliográficos, así como al Dr. Rogers McVaugh por hacernos partícipes del resultado de sus investigaciones acerca del nombre correcto de la especie de Sisyrinchium con flores azules.

\section{LITERATURA CITADA}

Beaman, J. H. 1965. A preliminary ecological study of the alpine flora of Popocatepetl and Iztaccihuatl. Bol. Soc. Bot. Méx. 29: 63-75.

Bicknell, E. P. 1900. Studies in Sisyrinchium VIII. Sisyrinchium californicum and related species of the neglected genus Hydastylus. Bull. Torr. Bot. Club 27: 273-387.

Cruden, R. W. 1971. The systematics of Rigidella (Iridaceae). Brittonia 23: 217-225. 
Foster, R. C. 1945. A revision of the North American species of Nemastylis. Contr. Gray Herb. 155: 26-44. Hemsley, W. B., 1879-1888. Botany, In: Godwin F.D. \& O. Salvin. Biologia Centrali-Americana. R.H. Porter. 3: 325-331. Londres.

Johnston, I. M. 1938. The species of Sisyrinchium in Uruguay, Paraguay and Brazil. Journ. Arn. Arb. 19: 376-401.

Matuda, E. 1962 (1961). Las Iridáceas del Valle de México y sus alrededores. An. Inst. Biol. Méx. 32: 157175.

Matuda, E. 1964. Las Iridáceas del Estado de México. Dirección de Recursos Naturales del Gobierno del Estado de México. Toluca, México. 19 pp.

Molseed, E. 1970. The genus Tigridia (Iridaceae) of Mexico and Central America. Univ. Calif. Publ. Bot. 54: 1-127.

Ravenna, P. F. 1965. Notas sobre Iridaceae. II. Bol. Soc. Arg. Bot. 4: 311-322.

Reiche, C. 1914. La vegetación en los alrededores de la capital de México. México, D.F. 143 pp.

Reiche, C. 1926. Flora excursoria en el Valle Central de México. Talleres Gráficos de la Nación, México, D. F. 303 pp.

Sánchez S., O. 1969. La Flora del Valle de México. Editorial Herrero, S.A. México, D.F. 519 pp.

Steyermark, J. A. 1948. Orthrosanthus chimboracensis and its varieties (Iridaceae). Lloydia 11: 14-20. 